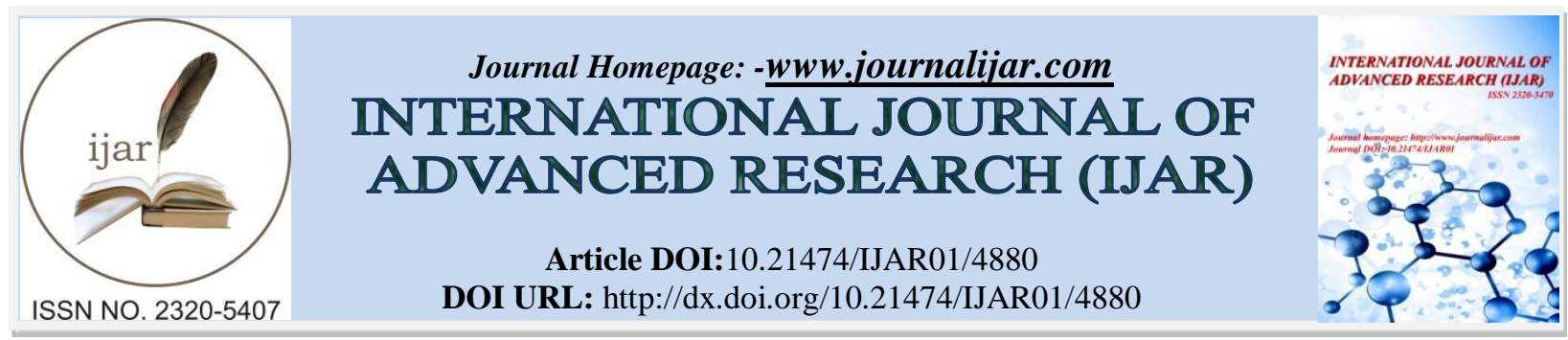

RESEARCH ARTICLE

\title{
CONTENT BASED IMAGE RETRIEVAL USING SVM ALGORITHM AND CONTOURLET TRANSFORM COEFFICIENTS DISTRIBUTION.
}

\author{
Satish Tunga ${ }^{1}$ and Jayadevappa $\mathrm{D}^{2}$. \\ 1. Dept. of Telecommunication Engineering, MSRIT, Bengaluru, \& Research Scholar, Jain Univ., Bengaluru, \\ India. \\ 2. Dept. of Electronics \& Inst. Engineering, JSS Academy of Technical Education, Bengaluru, VTU, Belgavi, \\ India.
}

\section{Manuscript Info}

\section{Manuscript History}

Received: 18 May 2017

Final Accepted: 20 June 2017

Published: July 2017

Key words:-

Content Based Image Retrieval Support

Vector MachineContourlet Transform.

\begin{abstract}
Conventional content-based image retrieval schemes may suffer from practical applications. Image databases are often composed of several groups of images and span very different scales in the space of lowlevel visual descriptors the interactive retrieval of such image classes is then very difficult. To address this challenge, we propose the support vector machine (SVM) algorithm with contourlet transform coefficients distribution. SVM is used to find out the optimal result and to evaluate the generalization ability under the limited training samples. It gives faster result as compared to other. An SVM classifier can be learned from training data of relevance images and irrelevance images marked by users. Features of the face image are extracted in the spectrum domain using contourlet Transform and this transform addresses the problem of representing the images with smooth contours in different directions by providing two additional properties which are directionality and anisotropy. This method will overcome the introduction of the noisy examples by the users. In the proposed technique, multiple feature distances are combined to obtain image similarity. The extensive experiments are performed on two different image data bases to validate the superiority of the proposed method.
\end{abstract}

Copy Right, IJAR, 2017,. All rights reserved.

\section{Introduction:-}

With the development of the Internet, and the availability of image capturing devices, the size of digital image collection is increasing rapidly. Efficient image searching, browsing and retrieval tools are required by users from various domains, including remote sensing, crime prevention, publishing, medicine etc. For this purpose, many general purpose image retrieval systems [1], [2] have been developed. Internet makes it possible for the human to access this huge amount of information. The greatest challenge of the World Wide Web is that, the more information available about a given topic, the more difficult it is to locate accurate and relevant information. Most users know what information they need, but are unsure where to find it. Search engines can facilitate the ability of users to locate such relevant information. Motivated by the limitations of the low-level based approach, an interactive learning mechanism was appeared in recent years [3], [4]. From another viewpoint, CBIR can also be considered as a search problem in the feature space to find more images similar to the query image. With the feedback technique, the available information is not only the feature space itself, but also the relevance information 
given by users. Therefore, we can build a classifier to separate two classes of relevance images and irrelevance images. Using the classifier model, we can retrieve much more images relevant to the query efficiently in the feature space. Based on SVM [5], [6], a classifier can be learned from training data of relevance images and irrelevance images marked by users. Then the model can be used to find more relevance images in the whole database.

Content Based Image Retrieval (CBIR) methods can be assigned to one of two major approaches, spatial or transform domain techniques. Spatial domain techniques are mostly based on color, shape, or texture features that are extracted directly from images. Transform domain methods utilize global information from images to perform image retrieval. The global information of an image is fundamentally represented by a small number of features derived from the spectrum of the image after transforming it from the special domain to the transform domain, using contourlet transform [7]. Contourlet Transform (CT) addresses the problem of representing the images with smooth contours in different directions, by providing two additional properties which are directionality and anisotropy, comparing with wavelets [8], [9]. The normalized standard deviation (SD), which is computed on each directional sub-band of the CT decomposed image, is used to form the image feature vector. These normalized feature vectors are used for the creation of the features database.

\section{Related Work:-}

The earliest use of the term content-based image retrieval in the literature seems to have been by Kato et.al. [10] to describe his experiments into automatic retrieval of images from a database by color and shape feature. The term has since been widely used to describe the process of retrieving desired images from a large collection on the basis of features such as color, texture and shape that can be automatically extracted from images themselves. Support Vector Machine (SVM) has been a promising method for data classification and regression [11], [12]. SVMs are a set of related supervised learning methods used for classification and regression [13], [14]. They belong to a family of generalized linear classifiers. In other terms, SVM is a classification and regression prediction tool that uses machine learning theory to maximize predictive accuracy while automatically avoiding over-fit to the data. Support Vector machines can be defined as systems which use hypothesis space of a linear functions in a high dimensional feature space, trained with a learning algorithm from optimization theory that implements a learning bias derived from statistical learning theory.

Support vector machine is supervised learning techniques that analyze data and identify pattern used for classification. It takes a set of input, read it and for each input desired output form [15]. This type of method is known as classification. There are two types of output. If output is discrete then classification performed and if output is continuous then regression performed.Image mining [16] is the main concept which can extract potential information from the collection. For color based image extraction RGB model is used, RGB component taken from each and every image. The top ranked images are further regrouped according to texture features. The gray level cooccurrence matrix (GLCM) used texture calculations (contrast, dissimilarity, homogeneity). Texture based classification is simply easy and efficient for real time applications as compared to Entropy method. The authors also evaluate the performance with the help of precision v/s recall graph. In Kun-Che.et al. [17] pixel wise image characteristics were extracted and changed into a database like table which permits a variety of data mining algorithms to make explorations on it. The performance of a CBIR system mainly depends on the particular image representation and similarity matching function employed [18]. CBIR or Content Based Image Retrieval is the retrieval of images based on visual features such as colour, texture and shape [19]. Reasons for its development are that in many large image databases, traditional methods of image indexing have proven to be insufficient, laborious, and extremely time consuming.

\section{Materials:-}

\section{Preprocessing:-}

Image contains lot of information which cannot be defined using text. Every image contains lot of information, using which lot of information can be mined. Various methods are proposed by researchers. In this method the images are preprocessed before they are stored in database. Then these images are clustered using various components of RGB model, after which the top ranked images are again clustered using support vector machine algorithm(SVM).This preprocessing enhances the image quality and removes the noise. Wavelets are good at isolating the discontinuities at edge points of an image, but cannot see the smoothness along the contours. Contourlet Transform (CT) addresses the problem of representing the images with smooth contours in different directions, by providing two additional properties which are directionality and anisotropy, comparing with wavelets [20].Contourlet transform is an extension of the wavelet transform which uses multi scale and directional filter 
banks. Although the wavelet transform is powerful in representing images containing smooth areas separated with edges. It cannot perform well when the edges are smooth curves. New developments in directional transforms, known as Contourlets. The Contourlet transform is one of the new geometrical image transforms, which represents images containing contours and textures. The contourlet transform effectively captures smooth contours images that are the dominant feature in natural images. Do and Vetterli [21] constructed a discrete domain multiresolution and multidirectional expansion using non-separable filter banks to obtain sparse expansions for typical images having smooth contours.

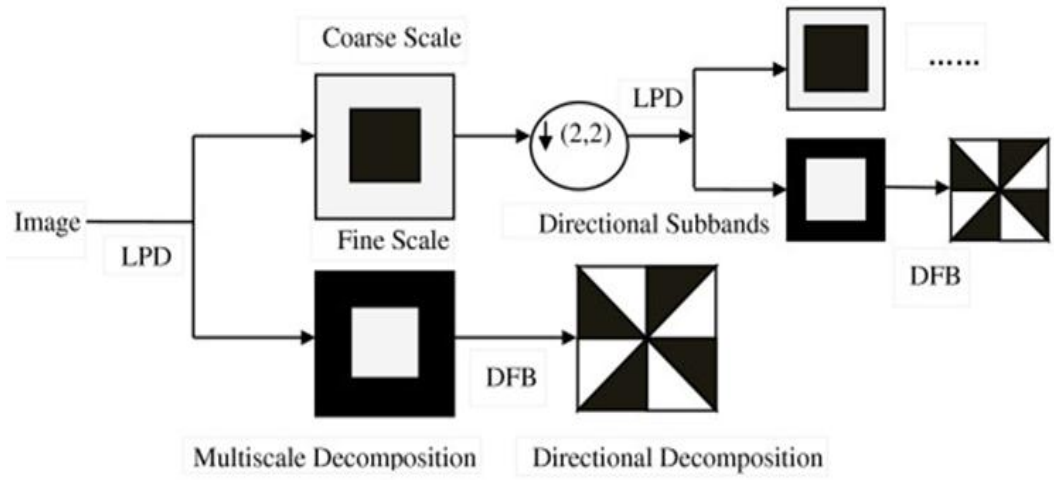

Figure 1:- Block diagram of Contourlet transform.

In contourlet transform, the directional information is preserved in each sub-band and is captured by computing its energy. This energy is capable of enhancing weak and complex boundaries in details. Further, due its directional image expansion property, smoothness along the contours can be easily achieved. Therefore, contourlet transforms are well suited for the further enhancement of the object boundary. The treatment of the proposed algorithm is divided into two stages. The first stage is used to compute the energy of the contourlet transform decomposed MR image. The second stage corresponds to integration of this energy using SVM.

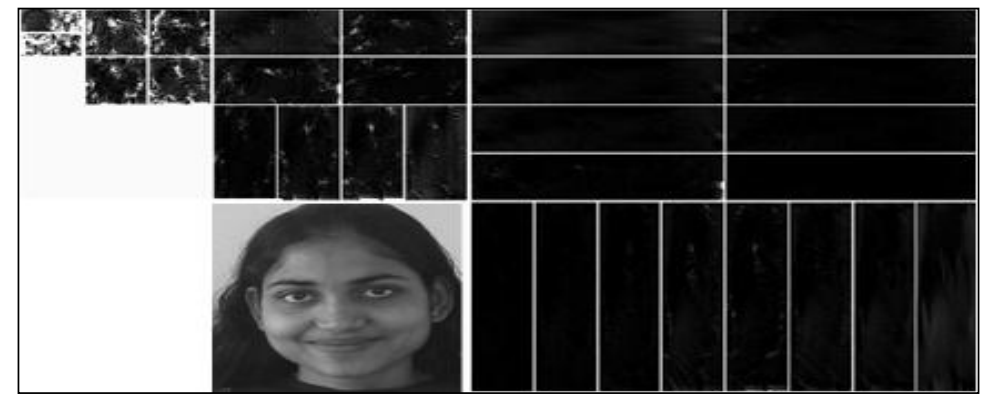

Figure 2:- Four levels Contourlet decomposition

\section{Support Vector machine:-}

In machine learning there are two types of methods supervised and unsupervised. Supervised learning based on learn by result and unsupervised based on learn by example. Supervised learning takes input as a set of training data. Support vector machine is a supervised learning technique that analyzes data and identify pattern used for classification. Support vector machine is used to constructs a hyperplane or set of hyperplane in a high or infinitedimensional space, which can be worn for classification, regression. A good separation is achieved by the hyperplane that has the largest distance to the nearest training data point of any class (functional margin), given that in general the larger the margin the lower the generalization error of the classifier. There are many hyperplane that might classify the data. It is mainly used to find maximum margin hyper planes in a high dimensional feature space. It divides the feature set into arbitrary groups and classifies them to return the best optimal result.

It views the given image database as two sets of vectors in an ' $n$ 'dimensional space and constructs a separating hyper plane that maximizes the margin between the images relevant to query and the images not relevant to the query. SVM is a kernel method and the kernel function used in SVM is very crucial in determining the performance. The basic principle of SVMs is a maximum margin classifier. Using the kernel methods, the data can be first 
implicitly mapped to a high dimensional kernel space. The maximum margin classifier is determined in the kernel space and the corresponding decision function in the original space can be non-linear [22]. The non-linear data in the feature space is classified into linear data in kernel space by the SVMs. This is illustrated in figure3.

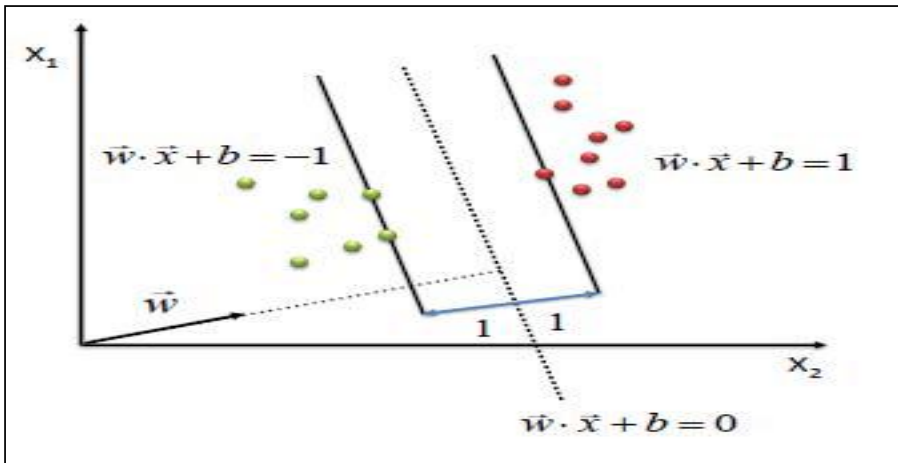

Figure 3:- Support vector machine model

The mathematically description of SVM is as follows:

Consider a given set of points in the form of training data which separate two classes of pattern based on given training set:

$$
S=\left\{\left(x_{1}, y_{1}\right),\left(x_{2}, y_{2}\right),\left(x_{3}, y_{3}\right) \ldots \ldots . .\left(x_{i}, y_{i}\right)\right\}
$$

Where, $x_{i}$ is a $p$-dimension real vectors, $y_{i}=\{-1,+1\}$ and $n$ is a no. of samples. According to Vapnik's equation,

$$
\begin{aligned}
& y_{i}=\leq w \cdot x \geq+b \\
& y_{i}=\sum w_{i} \cdot x_{i}+b
\end{aligned}
$$

where $w$ is a $p$-dimension vector and $b$ is constant or scalar.

By adding a scalar value $b$ it increases the margin between hyperplanes and in the absence of $b$ hyperplane is forced to pass through the origin. So in SVM we always use parallel hyperplanes which maintain distance between them. Parallel hyperplanes can be described by equation

$$
\begin{aligned}
& w \cdot x_{i}+b=1 \quad y_{i}=1 \\
& w \cdot x_{i}+b=-1 \quad y_{i}=-1
\end{aligned}
$$

It can select these hyper planes so that there are no points between them and then make an effort to maximize their distance. As we know in feature space there are number of hyper planes but choose the one for which the distance to the closest point is maximal is called optimal separating hyper planes. Since the distance to the closest point is 1. After subtracting the two distances we get the summed distance from separating hyper planes to the nearest points. During the process users can mark an image as either relevance or irrelevance.

\section{Methodology:-}

In this method the images are preprocessed before they are stored in database. This preprocessing enhances the image quality and removes the noise. Then these images are clustered using support vector machine algorithm (SVM). Then, the query image and the target images are compared using these features and the similar image is retrieved. The following diagram represents overall working of the proposed system.

The features such as edge and texture orientations are captured by using contourlet decomposition with a 4 level LP decomposition. At each level, the number of directional sub-bands are (3, 4, 8 and 16) respectively. For LP decomposition and directional sub-band decomposition the 'pkva' filters are used. These parameters results in a 32 dimensional feature vector The normalized standard deviation, which is computed on each directional sub-band of the CT decomposed image, is used to form the image feature vector. These normalized feature vectors are used for the creation of the features database. 
We consider a database created by transferring the images in the hard disk. The decomposition of the images will be done using contourlet transforms and stored in feature database module. By applying contourlet transform with different orientations and different scales on all the blocks of an image, we obtain a set of magnitudes for each block. We compare the standard deviation for each orientation and obtain feature for that block and subsequently a feature vector is obtained for the entire image. Thus, features are calculated for each and every image and the resulted feature vectors are stored in feature database.

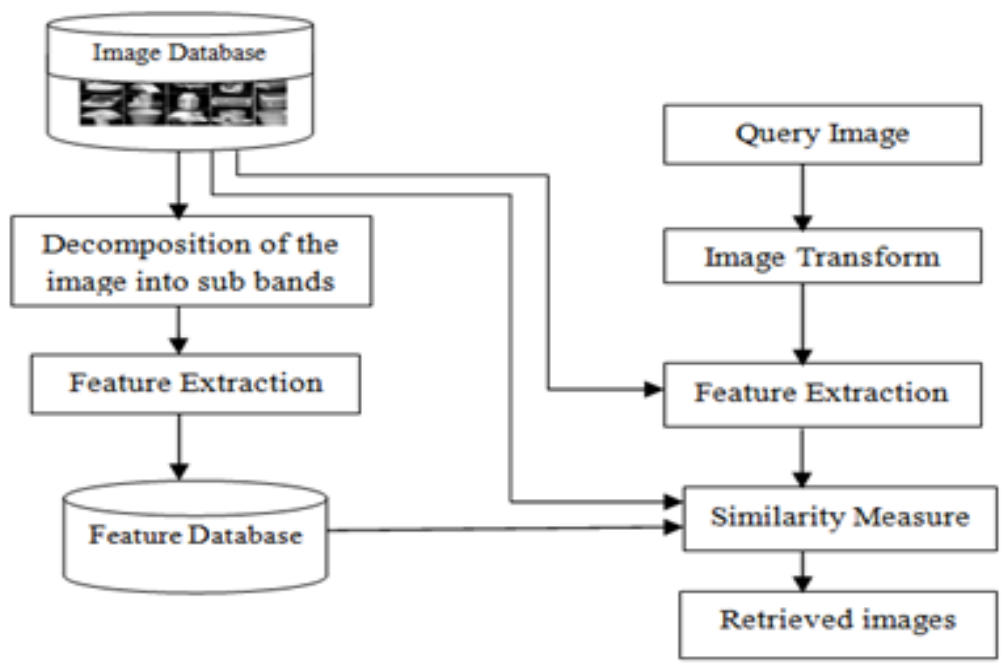

Figure 4:- Block diagram of the proposed CBIR system.

The feature vectors of images from all the classes are trained by SVM according to the label attached to the feature vector and label the query image with the class it belongs. Finally extract variables of the trained SVM for required class and perform classification of the images, and then indexed images are stored in the result folder.

\section{Results AndDiscussion:-}

For the performance analyses of the features selection methods two face images databases are used. They are COIL image database [23] and Georgia Tech (GT) face database [24]. The performance of the proposed system was checked by the following performance measures such as precision and recall. They are defined in terms of a set of retrieved images, such as the list of face images retrieved, and a set of the relevant images in the database (the list of all images on the database that belong to the same person). The standard definitions of these two measures are given by following equations.

$$
\begin{aligned}
& \text { Pr ecision }=\frac{\text { Number of relevant images retrieved }}{\text { Total number of images retrieved }} \\
& \operatorname{Re} \text { call }=\frac{\text { Number of relevant images retrieved }}{\text { Total number of relevant images retrieved in database }}
\end{aligned}
$$




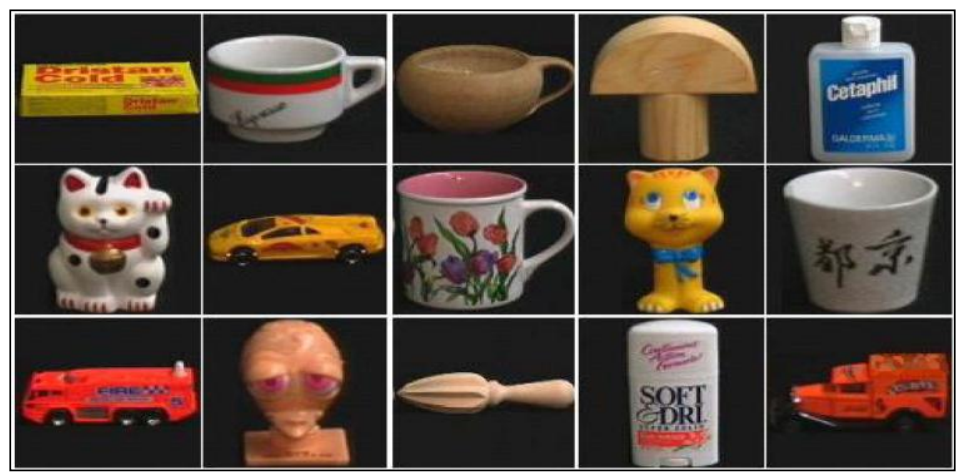

Figure 5:- Sample images from COIL image database.

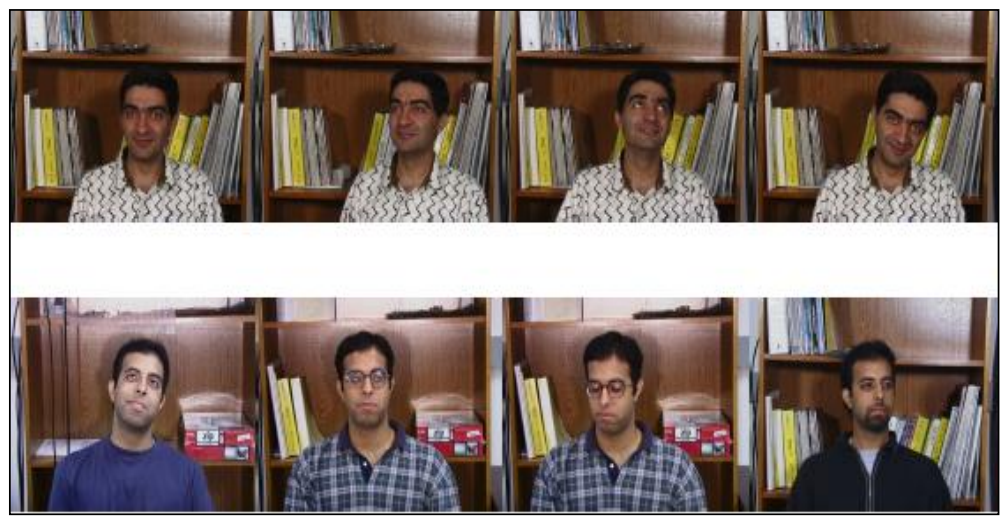

Figure 6:- Sample images from GT face image database.

Precision/recall values plotted against number of retrieved images for all proposed image retrieval techniques. Here SVM-Contoulet based image retrieval technique gives the highest precision/recall crossover values compare to curvelet and Gabor filter techniques specifying the best performance. The crossover point varies for different image category, and the highest crossover point 91 is achieved for white bottle and Tea cup image category.

The methods SVM-Contourlet magnitude and only Gabor Filter features were applied to the image database having 1000 images of COIL image database having 1080 images spread across 15 categories. The query and database image matching is done using SVM-Contourlet transform classifier. The average precision and average recall are computed by grouping the number of retrieved images sorted according to classification of database images with the query image.

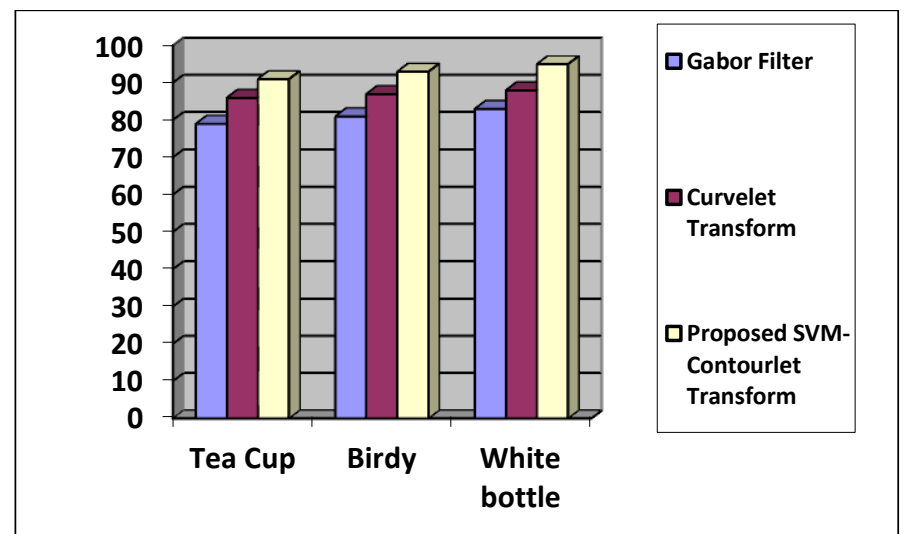

Figure 7:- Crossover points for Precision \& Recall plotted against number of retrieved images for different image categories on Coil Database. 
Table1 and Figure 8 shows the SVM-Contourlet produces the best retrieval rate comparing with Gabor filter and curvelet transform.

Table I:- Retrieval rate of CBIR for COIL Database

\begin{tabular}{|l|l|l|l|l|}
\hline \multirow{2}{*}{ Methods } & \multicolumn{4}{|l|}{ Retriving percentage of top match in the query image class } \\
\cline { 2 - 5 } & 5 of 15 & 8 of 15 & 10 of 15 & 15 of 15 \\
\hline Gabor filter [26] & 69.15 & 54.32 & 41.18 & 35.81 \\
\hline Curvelet transform [27] & 72.41 & 56.45 & 45.56 & 37.45 \\
\hline Proposed SVM-contourlet transform & 85.35 & 73.12 & 61.47 & 56.67 \\
\hline
\end{tabular}

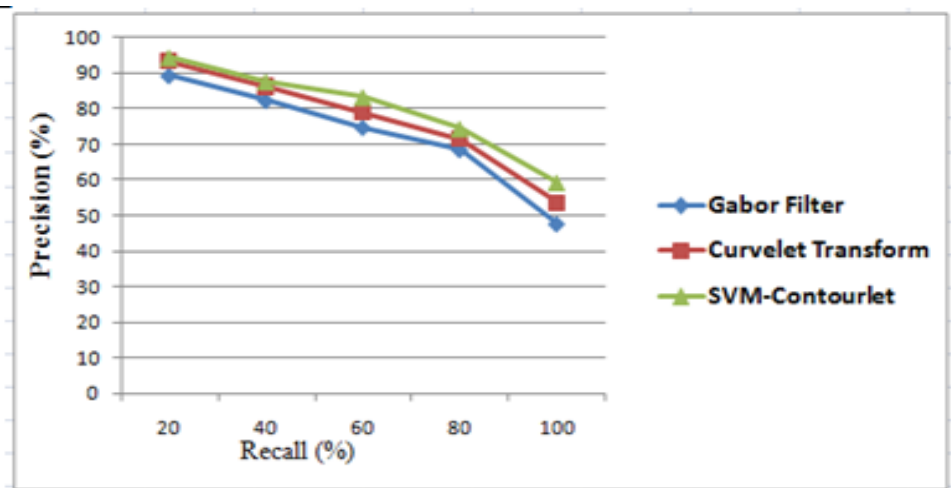

Figure 8:- Average retrieval rate verses number of Top matches of retrieval images for COIL Database.

Figure 8 and table II shows the results obtained using SVM-Contourlet based technique tested on COIL image database. There is significant improvement in results using SVM-Contourlet technique. The precision-recall curve shows crossover point for Gabor filter, Curvelet and proposed SVM-Contourlet method respectively. The average retrieval result of 1792 queries of GT image Database was tested by applying Curvelet Gabor Filter, Curvelet transform and SVM-Contourlet transform.

Table II:- Retrieval Accuracy of CBIR for COIL Database

\begin{tabular}{|l|l|l|l|}
\hline \multirow{2}{*}{ Recall (\%) } & Precison (\%) & \multicolumn{2}{|l|}{} \\
\cline { 2 - 4 } & Gabor Filter & Curvelet Transform & SVM-Contourlet Transform \\
\hline 20 & 89.35 & 93.34 & 94.34 \\
\hline 40 & 82.34 & 86.15 & 87.56 \\
\hline 60 & 74.76 & 79.01 & 83.21 \\
\hline 80 & 68.56 & 71.56 & 74.32 \\
\hline 100 & 47.67 & 53.57 & 59.21 \\
\hline
\end{tabular}

The following figure 9 illustrates the percentages of precesion verses recall for GT face database for the respective techniques.

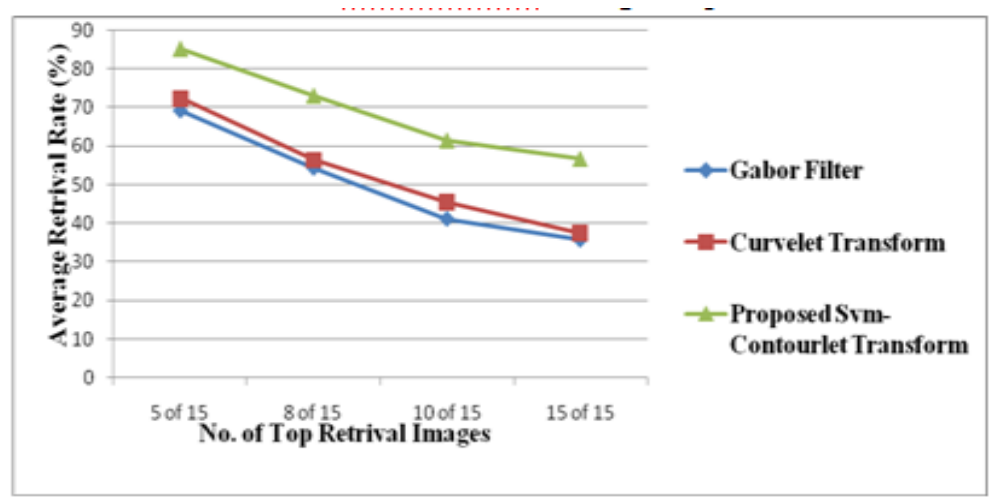

Figure 9:- Percentages of precision verses recall for GT face database. 


\section{Conclusion:-}

This proposed work presented SVM technique using Contourlet transform for image retrieval framework. It uses composite learning method using SVM and Contourlet transform. In this technique, multiple feature distances are combined to obtain image similarity. The extensive experiments are performed on COIL image and GT Face Database to validate the superiority of the proposed method. This framework has demonstrated very promising retrieval accuracy as compared to the existing systems. The performance of the proposed system is compared with the curvelet and Gabor filter techniques.

\section{References :-}

1. Y. Liu, et al., "A survey of content based image retrieval with high-level semantics," Journal of Pattern Recognition,

2. vol. 40, pp. 262-282, Nov. 2007.

3. SatishTunga et al., "A Comparative Study of Content Based Image Retrieval Trends and Approaches," International Journal of Image Processing, Volume (9), Issue (3), 2015.

4. S. Belongie, et al., "Shape Matching and Object Recognition Using Shape Contexts," IEEE Trans. Pattern Analysis and Machine Intelligence, 24(4):509-522, 2002.

5. Y. Chen, et al., "A Region-Based Fuzzy Feature Matching Approach to Content-Based Image Retrieval," IEEE Trans.

6. Pattern Analysis and Machine Intelligence, 24(9):252-1267, 2002.

7. C. Cortes, et al., "Support-Vector Networks", Machine Learning, 20, pp. 273-297, 1995.

8. C.J.C. Burges, "A Tutorial on Support Vector Machines for Pattern Recognition”, Data Mining and Knowledge

9. Discovery, 2(2), pp. 1-47, 1998.

10. M. N. Do and M. Vetterli, "The Contourlet Transform: An Efficient Directional Multiresolution Image

11. Representation," IEEE Transactions on Image Processing, Vol. 14, No. 12, 2005.

12. Lamard et al., "Content Based Image Retrieval based on Wavelet Transform Coefficients Distribution," Proceedings

13. of the 29th Annual International Conference of the IEEE EMBSCitéInternationale, Lyon, France, August 23-26,

14. 2007.

15. Rao C. S et al., "Content Based Image Retrieval using Contourlet Transform," GVIP Journal, Vol 7, Issue 3,

16. November 2007.

17. Hirata K. and Kato T. "Query by visual example content-based image retrieval", In Proc. of Third International

18. Conference on Extending Database Technology, EDBT'92, 1992, pp 56-71.

19. Chang and J. Lin., "Training nu-support vector classifiers: Theory and algorithms," Neural Computation, 13:2119-

20. 2147, 2001.

21. H. Yu, J. Han, and K. C. Chang., "PEBL: Positive-example based learning for Web page classification using SVM,"

22. In Proc. 8th Int. Conf. Knowledge Discovery and Data Mining (KDD'02), pages 239-248, Edmonton, Canada,

23. 2002 .

24. S. Tong and E. Chang. "Support vector machine active learning for image retrieval," In Proceedings of the ninth

25. ACM International conference on Multimedia, pages 107\{118. ACM Press, 2001.

26. Xiang-Yang et al., "Active SVM-based relevance feedback using multiple classifiers ensemble and features

27. Reweighting," Engineering Applications of Artificial Intelligence 26, 368-381, Elsevier Ltd, 2013.

28. Patheja P.S, et al., "An Enhanced Approach for Content Based Image Retrieval", International Science Congress

29. Association, Research Journal of Recent Sciences, ISSN 2277-2502, Vol. 1(ISC-2011), 415-418, 2012.

30. Kannan,Dr.V.Mohan,Dr.N.Anbazhagan,"Image Clustering and Retrieval using Image Mining Techniques," IEEE

31. International Conference on Computational Intelligence and Computing Research, 2010.

32. Kun-Che Lu and Don-Lin Yang, "Image Processing and Image Mining using Decision Trees," Journal of

33. Information Science and Engineering 25, 989-1003, 2009.

34. M.E. ElAlami, "A new matching strategy for content based image retrievalsystem," Applied soft computing, 14,

35. 2014.

36. Guang-Hai Liu, Jing-YuYang, "Content-based image retrieval using color difference histogram," Pattern

37. Recognition, 46, 2013. 
38. M. N. Do and M. Vetterli, "The Contourlet Transform: An Efficient Directional Multiresolution Image

39. Representation," IEEE Transactions on Image Processing, vol. 14, No. 12, 2005.

40. M. N. Do and M. Vetterli, "Framing Pyramids", IEEE, Transactions on Signal Processing, Vol. 51, No. 9, pp.

41. 2329-2342, 2003.

42. K. K. Seo, "An application of one-class support vector machines in content based image retrieval," Expert Systems

43. with Applications, Vol. 33(2), pp.491-498, 2007.

44. http://www.cs.columbia.eduiCAVE/software/softlib/coil-100.php.

45. Georgia Tech (GT) Face Database Available at. ftp://ftp.ee.gatech.edu./pub/users/hayesfacedb.

46. Indian face database website: http://viswww. cs.umass.edu/ vidit/IndianFaceDatabaseIIT, Technology, Kanpur.

47. Sultan A, et al., "Classification of Image Database using SVM Gabor Magnitude," IEEE 2012 International

48. Conference on Multimedia Computing and Systems (ICMCS), Tangeiers, Morocco.

49. L. Ni and H. C. Leng, "Curvelet Transform and Its Application in Image Retrieval," $3^{\text {rd }}$ International Symposium on

50. Multispectral Processing and Pattern Recognition, Proceedings of SPIE, Vol. 5286, 2013. 